\title{
Optimal Stochastic Linearization for Range-Based Localization
}

\author{
Frederik Beutler, Marco F. Huber, and Uwe D. Hanebeck
}

\begin{abstract}
In range-based localization, the trajectory of a mobile object is estimated based on noisy range measurements between the object and known landmarks. In order to deal with this uncertain information, a Bayesian state estimator is presented, which exploits optimal stochastic linearization. Compared to standard state estimators like the Extended or Unscented Kalman Filter, where a point-based Gaussian approximation is used, the proposed approach considers the entire Gaussian density for linearization. By employing the common assumption that the state and measurements are jointly Gaussian, the linearization can be calculated in closed form and thus analytic expressions for the range-based localization problem can be derived.
\end{abstract}

\section{INTRODUCTION}

In applications such as mobile robot navigation or telepresence, the position of a moving object is often localized based on range measurements between the object and known landmarks. These ranges can for example be measured by times of arrival or field strengths [1].

Existing range-based localization algorithms can be divided into two classes. Approaches of the first class assume exact (or almost exact) range measurements. As long as this assumption is satisfied, closed-form localization approaches as those in [2]-[6], gradient descent algorithms, or methods based on linearization via Taylor-series expansion perform very well. However, these approaches merely allow for a static localization, i.e., a separate localization is performed at every time step. Furthermore, accurate range measurements require specialized and expensive hardware.

Dealing with inaccurate measurements that may arise for example from signal strength information or ultrasonic range finders requires range-based localization approaches from the second class. Based on probabilistic models that capture measurement uncertainties, the object's position and velocity can be estimated by means of a Bayesian estimator in a recursive fashion. This allows for dynamic localization, i.e., the combination of dead reckoning and static localization, for a smoother and more robust localization.

Generally, a closed-form evaluation of the equations of the Bayesian estimator is not possible due to nonlinearities in the measurement model and the object's dynamics model. Thus, approximate estimators like the Extended Kalman Filter

F. Beutler and U. D. Hanebeck are with the Intelligent Sensor-ActuatorSystems Laboratory (ISAS), Institute for Anthropomatics, Karlsruhe Institute of Technology (KIT), Karlsruhe, Germany. beut lere i eee. org, uwe. hanebeck@ieee. org.

Marco F. Huber is with the Variable Image Acquisition and Processing Research Group, Fraunhofer Institute of Optronics, System Technologies and Image Exploitation IOSB, Karlsruhe, Germany. marco.huberdieee.org.
(EKF) [7] or the Unscented Kalman Filter (UKF) [8] are typically employed, particularly for range-based localization.

In this paper, we proposed an analytically solvable estimator. For this purpose, the standard measurement model consisting of the Euclidean norm and additive noise is slightly modified by moving the noise into the Euclidean norm and by considering squared ranges. Based on this modification, we derive an analytic expression of the first two moments, i.e., mean and covariance, characterizing the object's position and velocity estimates. This analytic moment calculation (AMC) can be considered as Gaussian estimation employing stochastic linearization. In contrast to the point-based Gaussian estimators such as the EKF or the UKF, the proposed AMC algorithm considers the entire Gaussian density for linearization, leading to more accurate localization results.

The structure of the paper is as follows. The problem formulation in Sec. II provides the modified measurement model and the object's dynamics model. In Sec. III, the general form of the proposed state estimator is described. Based on the modified measurement model, the moments can be calculated in closed form, which is shown in Sec. IV. In Sec. V, the proposed algorithm is compared with the EKF, the UKF, and a closed-form solution via simulations and experiments. Conclusions and an outlook to future work are given in Sec. VI.

\section{PROBLEM Formulation}

In this paper, dynamic localization of a mobile object is considered. The dynamic state of the object is described by means of the state vector $\underline{\boldsymbol{x}}_{k}=\left[\underline{\boldsymbol{x}}_{k, P}^{\mathrm{T}}, \underline{\boldsymbol{x}}_{k, V}^{\mathrm{T}}\right]^{\mathrm{T}}$ consisting of the object's position $\underline{\boldsymbol{x}}_{k, P} \in \mathbb{R}^{3}$ and velocity $\underline{\boldsymbol{x}}_{k, V} \in \mathbb{R}^{3}$ in three-dimensional space. Here, $k=0,1, \ldots$ is the discrete time index.

\section{A. Dynamics Model}

The dynamic behavior - the motion - of the object is described by means of the linear discrete-time dynamic system

$$
\underline{\boldsymbol{x}}_{k+1}=\mathbf{A} \cdot \underline{\boldsymbol{x}}_{k}+\underline{\boldsymbol{w}}_{k},
$$

where the noise $\underline{\boldsymbol{w}}_{k}$ is assumed to be zero-mean white Gaussian. For a position velocity model [9], the matrix A and the covariance of the process noise $\mathbf{C}^{w}$ are given by

$$
\mathbf{A}=\left[\begin{array}{cc}
\mathbf{I} & T \cdot \mathbf{I} \\
\mathbf{0} & \mathbf{I}
\end{array}\right], \mathbf{C}^{w}=\left[\begin{array}{cc}
\frac{T^{3}}{3} \mathbf{C}_{c}^{w} & \frac{T^{2}}{2} \mathbf{C}_{c}^{w} \\
\frac{T^{2}}{2} \mathbf{C}_{c}^{w} & T \cdot \mathbf{C}_{c}^{w}
\end{array}\right],
$$

where $T$ is sampling time and $\mathbf{I}$ is the identity matrix of adequate dimension. $\mathbf{C}_{c}^{w}$ is the process noise of the 
covariance from the continuous time system model $\mathbf{C}_{c}^{w}=$ $\operatorname{diag}\left(\left[\begin{array}{lll}C_{c, x}^{w} & C_{c, y}^{w} & C_{c, z}^{w}\end{array}\right]\right)$, where $C_{c, \xi}^{w}$ is the variance of dimension $\xi \in\{x, y, z\}$.

\section{B. Measurement Models}

For improving the object's state estimate, range measurements to $N$ landmarks at the known positions $\underline{S}_{i} \in \mathbb{R}^{3}$ with $i=1, \ldots, N$ are incorporated. The nonlinear relation between the object position and the landmark position is given by

$$
\boldsymbol{r}_{k, i}=\left\|\underline{S}_{i}-\underline{\boldsymbol{x}}_{k, P}\right\|_{2},
$$

where $\boldsymbol{r}_{k, i}$ is the Euclidean distance between object and landmark. $\|.\|_{2}$ is the Euclidean norm.

In a real scenario, the ranges cannot be measured exactly, i.e., measurement uncertainty has to be considered, which is usually done by incorporating a noise process into (2). Two possibilities arise for incorporation. In the first case given by

$$
\boldsymbol{r}_{k, i}=\left\|\underline{S}_{i}-\underline{\boldsymbol{x}}_{k, P}\right\|_{2}+\boldsymbol{v}_{k, i},
$$

the noise process $\boldsymbol{v}_{k, i}$ directly affects the range $\boldsymbol{r}_{k, i}$, which is the standard model. In the second case

$$
\boldsymbol{r}_{k, i}=\left\|\underline{S}_{i}-\underline{\boldsymbol{x}}_{k, P}-\underline{\boldsymbol{v}}_{k, i}\right\|_{2},
$$

which is called noise before non-linearity [10], the noise process affects the difference between object and landmark position. This measurement model can be interpreted in such a way that the positions of the landmarks are uncertain. In both measurement models, the noise process is assumed to be zero-mean white Gaussian.

In this paper, we will focus on the second model (4) mainly for two reasons. First, the standard model (3) is only appropriate in situations where the distance $\boldsymbol{r}_{k, i}$ is large compared to the variance of the noise $\boldsymbol{v}_{k, i}$. Otherwise, negative ranges are possible, which is not true in reality. This problem cannot occur in the second measurement model. Second, the model in (4) allows analytic moment calculation as will be shown in the following.

\section{RECURSIVE STATE Estimation}

Both the measurement model (4) and the system model (1) are utilized in a Bayesian estimation framework for recursively estimating the state $\underline{\boldsymbol{x}}_{k}$. For this purpose, two alternating steps, i.e., prediction and filtering, are performed.

\section{A. Prediction Step}

In the prediction step, we are interested in calculating the predicted mean $\underline{\mu}_{k+1}^{p}$ and covariance $\mathbf{C}_{k+1}^{p}$ of the state. Thanks to the linear system model (1), the prediction can be performed in closed form by means of the prediction step of the Kalman filter. Assuming that the result of the previous filter step is Gaussian and thus given by the density function $f^{e}\left(\underline{x}_{k}\right):=\mathcal{N}\left(\underline{x}_{k}-\underline{\mu}_{k}^{e}, \mathbf{C}_{k}^{e}\right)$, the mean and the covariance of the predicted density $f^{p}\left(\underline{x}_{k+1}\right):=\mathcal{N}\left(\underline{x}_{k+1}-\underline{\mu}_{k+1}^{p}, \mathbf{C}_{k+1}^{p}\right)$ are given by

$$
\underline{\mu}_{k+1}^{p}=\mathbf{A} \cdot \underline{\mu}_{k}^{e}, \mathbf{C}_{k+1}^{p}=\mathbf{A} \cdot \mathbf{C}_{k}^{e} \cdot \mathbf{A}^{\mathrm{T}}+\mathbf{C}^{w}
$$

It is worth mentioning that the approach proposed in this paper is not restricted to linear system models. The techniques derived in the following for the filter step can also be used for special types of nonlinear dynamics. If the system model consists of a linear combination of trigonometric functions and/or polynomials, as it is the case for example in differential drive or bicycle kinematics, an analytic calculation of the predicted mean and covariance is still possible.

\section{B. Filter Step}

In the filter step, the current range measurement $\underline{\hat{r}}_{k}=$ $\left[\hat{r}_{k, 1}, \ldots, \hat{r}_{k, N}\right]^{\mathrm{T}}$ is used for updating the result of the prediction step $f^{p}\left(\underline{x}_{k}\right)$ according to Bayes' rule

$$
f^{e}\left(\underline{x}_{k}\right)=c_{k} \cdot f\left(\underline{\hat{r}}_{k} \mid \underline{x}_{k}\right) \cdot f^{p}\left(\underline{x}_{k}\right),
$$

where $c_{k}=1 / \int f\left(\underline{\hat{r}}_{k} \mid \underline{x}_{k}\right) \cdot f^{p}\left(\underline{x}_{k}\right) \mathrm{d} \underline{x}_{k}$ is a normalization constant and $f\left(\underline{\hat{r}}_{k} \mid \underline{x}_{k}\right)$ is the likelihood defined by (4). Here, $\underline{\hat{r}}_{k}$ is a realization of the random vector $\underline{\boldsymbol{r}}_{k}$.

Generally, for nonlinear measurement models, a closedform calculation of the density $f^{e}\left(\underline{x}_{k}\right)$ as well as the mean $\mu_{k}^{e}$ and covariance $\mathbf{C}_{k}^{e}$ is not possible. Instead, appropriate approximations have to be applied. But for the special measurement model in (4), mean $\underline{\mu}_{k}^{e}$ and covariance $\mathbf{C}_{k}^{e}$ can be calculated analytically if we assume that state and measurement are jointly Gaussian ${ }^{1}$. This assumption is only true if there is a linear relationship between $\underline{\boldsymbol{x}}_{k}$ and $\underline{\boldsymbol{r}}_{k}$. Otherwise, it is an approximation and corresponds to a special type of linearization. While typically applied linearization techniques like first-order Taylor-series expansion or unscented transformation [8], which are merely pointbased, our approach considers the entire predicted density. In doing so, it is possible to calculate the mean $\underline{\mu}_{k}^{e}$ and the covariance $\mathbf{C}_{k}^{e}$ according to

$$
\begin{aligned}
\mu_{k}^{e} & =\underline{\mu}_{k}^{p}+\mathbf{C}_{k}^{x, r} \cdot\left(\mathbf{C}_{k}^{r}\right)^{-1} \cdot\left(\underline{\hat{r}}_{k}-\underline{\mu}_{k}^{r}\right), \\
\mathbf{C}_{k}^{e} & =\mathbf{C}_{k}^{p}-\mathbf{C}_{k}^{x, r} \cdot\left(\mathbf{C}_{k}^{r}\right)^{-1} \cdot\left(\mathbf{C}_{k}^{x, r}\right)^{\mathrm{T}},
\end{aligned}
$$

where $\underline{\mu}_{k}^{r}$ is the predicted measurement value, $\mathbf{C}_{k}^{r}$ is the covariance of the predicted measurement and $\mathbf{C}_{k}^{x, r}$ is the cross-covariance between state and range measurement. In the following, an analytic calculation of these quantities is provided in order to allow for the closed-form evaluation of (5). For the rest of the paper, the time index $k$ and the superscripts at the symbol $f$ of density functions are omitted for brevity.

\section{Analytic Moment Calculation (AMC)}

\section{A. Modified Measurement Equation}

In order to obtain an analytic expression for the required quantities in (5), the range-based measurement equation (4) is squared, which results in

$$
\begin{aligned}
\boldsymbol{d}_{i}:=\left(\boldsymbol{r}_{i}\right)^{2}= & \left(\underline{S}_{i}-\underline{\boldsymbol{x}}_{P}\right)^{\mathrm{T}} \cdot\left(\underline{S}_{i}-\underline{\boldsymbol{x}}_{P}\right) \\
& -2 \cdot\left(\underline{S}_{i}-\underline{\boldsymbol{x}}_{P}\right)^{\mathrm{T}} \cdot \underline{\boldsymbol{v}}_{i}+\underline{\boldsymbol{v}}_{i}^{\mathrm{T}} \cdot \underline{\boldsymbol{v}}_{i},
\end{aligned}
$$

\footnotetext{
${ }^{1}$ This assumption is common in Gaussian filter like the EKF or the UKF.
} 
where $\boldsymbol{d}_{i}$ is a squared range assumed to be measured by $\hat{d}_{i}=\hat{r}_{i}^{2}$. The modified measurement equation (6) is then given for a single measurement in short term by

$$
\boldsymbol{d}_{i}=h_{i}\left(\underline{\boldsymbol{x}}_{P}, \underline{\boldsymbol{v}}_{i}\right)
$$

and for all measurements by

$$
\underline{\boldsymbol{d}}=\underline{h}\left(\underline{\boldsymbol{x}}_{P}, \underline{\boldsymbol{v}}\right) \text {. }
$$

It is important to note that the moments of the squared ranges (indicated with superscript $d$ ) in contrast to normal range measurements (indicated with superscript $r$ ) have to be used in (5), which leads to

$$
\begin{aligned}
\underline{\mu}^{e} & =\underline{\mu}^{p}+\mathbf{C}^{x, d} \cdot\left(\mathbf{C}^{d}\right)^{-1} \cdot\left(\underline{\hat{d}}-\underline{\mu}^{d}\right) \\
\mathbf{C}^{e} & =\mathbf{C}^{p}-\mathbf{C}^{x, d} \cdot\left(\mathbf{C}^{d}\right)^{-1} \cdot\left(\mathbf{C}^{x, d}\right)^{\mathrm{T}} .
\end{aligned}
$$

\section{B. Moment Calculation}

Based on (8), the moments $\mu^{d}, \mathbf{C}^{d}$, and $\mathbf{C}^{x, d}$ of the squared range measurement $\underline{\boldsymbol{d}}$ can be calculated in closed form, if the random vectors $\underline{\boldsymbol{x}}$ and $\underline{\boldsymbol{v}}$ are assumed as stemming from Gaussians $\mathcal{N}\left(\underline{x}-\mu^{p}, \overline{\mathbf{C}}^{p}\right)$ and $\mathcal{N}\left(\underline{v}, \mathbf{C}^{v}\right)$. The mean and covariance of the state are given by

$$
\underline{\mu}^{p}=\left[\begin{array}{c}
\underline{\mu}^{x_{P}} \\
\underline{\mu}^{x_{V}}
\end{array}\right], \mathbf{C}^{p}=\left[\begin{array}{cc}
\mathbf{C}^{x_{P}} & \mathbf{C}^{x_{P}, x_{V}} \\
\mathbf{C}^{x_{V}, x_{P}} & \mathbf{C}^{x_{V}}
\end{array}\right],
$$

where the state consists of the position and the velocity. The measurement noise $\underline{v}$ is zero-mean with a covariance of

$$
\mathbf{C}^{v}=\left[\begin{array}{ccccc}
\mathbf{C}_{1}^{v} & \ldots & \mathbf{C}_{1, j}^{v} & \ldots & \mathbf{C}_{1, N}^{v} \\
\vdots & \vdots & \vdots & \vdots & \vdots \\
\mathbf{C}_{i, 1}^{v} & \ldots & \mathbf{C}_{i, j}^{v} & \ldots & \mathbf{C}_{i, N}^{v} \\
\vdots & \vdots & \vdots & \vdots & \vdots \\
\mathbf{C}_{N, 1}^{v} & \ldots & \mathbf{C}_{N, j}^{v} & \ldots & \mathbf{C}_{N}^{v}
\end{array}\right]
$$

where $\mathbf{C}_{i, j}^{v}$ is the three-by-three-dimensional covariance matrix between the $i$ th and $j$ th landmark. It is worth mentioning that the landmarks are assumed to be spatially correlated, which allows general applicability of the proposed algorithm. Assuming uncorrelatedness would simplify the following derivations.

1) Mean: For calculating the mean, it can be utilized that

$$
\begin{aligned}
\underline{\mu}^{d}=\mathrm{E}_{\underline{\boldsymbol{d}}}\{\underline{\boldsymbol{d}}\} & =\int \underline{d} \cdot f(\underline{d}) \mathrm{d} \underline{d} \\
& =\iiint \underline{d} \cdot f(\underline{x}, \underline{d}, \underline{v}) \mathrm{d} \underline{x} \underline{\mathrm{d}} \underline{d} \underline{\mathrm{d}} \underline{v}
\end{aligned}
$$

holds [11]. With (8) and Bayes' rule, the joint density function $f(\underline{x}, \underline{d}, \underline{v})$ of $\underline{\boldsymbol{x}}, \underline{\boldsymbol{d}}$ and $\underline{\boldsymbol{v}}$ can be written according to

$$
\begin{aligned}
f(\underline{x}, \underline{d}, \underline{v}) & =f(\underline{d} \mid \underline{x}, \underline{v}) \cdot f(\underline{x}, \underline{v}) \\
& =\delta(\underline{d}-\underline{h}(\underline{x}, \underline{v})) \cdot f(\underline{x}, \underline{v}),
\end{aligned}
$$

where $\delta(\underline{x}-\underline{\mu})$ is the Dirac delta distribution at position $\underline{\mu}$. As white measurement noise is assumed, the random vectors $\underline{\boldsymbol{x}}$ and $\underline{\boldsymbol{v}}$ are independent and thus, the joint density $f(\underline{x}, \underline{v})$ is given by

$$
f(\underline{x}, \underline{v})=f(\underline{x}) \cdot f(\underline{v}) .
$$

Plugging (11) into (10) and utilizing the sifting property of the Dirac delta distribution results in

$$
\begin{aligned}
& \underline{\mu}^{d}=\mathrm{E}_{\underline{\boldsymbol{x}}, \underline{\boldsymbol{v}}}\left\{\underline{h}\left(\underline{\boldsymbol{x}}_{P}, \underline{\boldsymbol{v}}\right)\right\} \\
& =\iint \underline{h}\left(\underline{x}_{P}, \underline{v}\right) \cdot f(\underline{x}) \cdot f(\underline{v}) \mathrm{d} \underline{x} \mathrm{~d} \underline{v} \\
& =\iint \underline{h}\left(\underline{x}_{P}, \underline{v}\right) \cdot f\left(\underline{x}_{P}\right) \cdot f(\underline{v}) \mathrm{d} \underline{x}_{P} \mathrm{~d} \underline{v} \\
& =\mathrm{E}_{\underline{\boldsymbol{x}}_{P}, \underline{\boldsymbol{v}}}\left\{\underline{h}\left(\underline{\boldsymbol{x}}_{P}, \underline{\boldsymbol{v}}\right)\right\} \text {, }
\end{aligned}
$$

where the velocity $\underline{x}_{V}$ is marginalized, because the variable $\underline{x}_{V}$ has no influence on the mean. Hence, the mean of $\underline{\boldsymbol{d}}$ can be calculated directly based on the nonlinear function $\underline{h}(\cdot)$ and the density of $\underline{\boldsymbol{x}}_{P}$ and $f(\underline{v})$. By using (7) in (12), the predicted measurement $\mu_{i}^{d}$ for the $i$ th range between landmark $i$ and the target is given by

$$
\begin{aligned}
\mu_{i}^{d}= & \mathrm{E}_{\underline{\boldsymbol{x}}_{P}, \underline{\boldsymbol{v}}}\left\{h_{i}\left(\underline{\boldsymbol{x}}_{P}, \underline{\boldsymbol{v}}\right)\right\} \\
= & \mathrm{E}_{\underline{\boldsymbol{x}}_{P}}\left\{\left(\underline{S}_{i}-\underline{\boldsymbol{x}}_{P}\right)^{\mathrm{T}} \cdot\left(\underline{S}_{i}-\underline{\boldsymbol{x}}_{P}\right)\right\} \\
& -2 \cdot \mathrm{E}_{\underline{\boldsymbol{x}}_{P}, \underline{\boldsymbol{v}}_{i}}\left\{\left(\underline{S}_{i}-\underline{\boldsymbol{x}}_{P}\right)^{\mathrm{T}} \cdot \underline{\boldsymbol{v}}_{i}\right\}+\mathrm{E}_{\underline{\boldsymbol{v}}_{i}}\left\{\underline{\boldsymbol{v}}_{i}^{\mathrm{T}} \cdot \underline{\boldsymbol{v}}_{i}\right\} \\
= & \left(\underline{S}_{i}-\underline{\mu}^{x_{P}}\right)^{\mathrm{T}} \cdot\left(\underline{S}_{i}-\underline{\mu}^{x_{P}}\right)+\operatorname{trace}\left(\mathbf{C}^{x_{P}}\right)+\operatorname{trace}\left(\mathbf{C}_{i}^{v}\right) .
\end{aligned}
$$

2) Covariance: For calculating the covariance $\mathbf{C}^{d}$ of the measurement process, it can be utilized that

$$
\begin{aligned}
\mathbf{C}^{d}= & \mathrm{E}_{\underline{\boldsymbol{d}}}\left\{\left(\underline{\boldsymbol{d}}-\underline{\mu}^{d}\right) \cdot\left(\underline{\boldsymbol{d}}-\underline{\mu}^{d}\right)^{\mathrm{T}}\right\} \\
= & \iiint\left(\underline{d}-\underline{\mu}^{d}\right) \cdot\left(\underline{d}-\underline{\mu}^{d}\right)^{\mathrm{T}} \cdot f\left(\underline{x}_{P}, \underline{v}, \underline{d}\right) \mathrm{d} \underline{x}_{P} \mathrm{~d} \underline{v} \mathrm{~d} \underline{d} \\
= & \iint \underline{h}\left(\underline{x}_{P}, \underline{v}\right) \cdot \underline{h}\left(\underline{x}_{P}, \underline{v}\right)^{\mathrm{T}} \cdot f\left(\underline{x}_{P}\right) \cdot f(\underline{v}) \mathrm{d} \underline{x}_{P} \mathrm{~d} \underline{v} \\
& \quad-\underline{\mu}^{d} \cdot\left(\underline{\mu}^{d}\right)^{\mathrm{T}} \\
= & \mathrm{E}_{\underline{\boldsymbol{x}}_{P}, \underline{\boldsymbol{v}}}\left\{\underline{h}\left(\underline{\boldsymbol{x}}_{P}, \underline{\boldsymbol{v}}\right) \cdot \underline{h}\left(\underline{\boldsymbol{x}}_{P}, \underline{\boldsymbol{v}}\right)^{\mathrm{T}}\right\}-\underline{\mu}^{d} \cdot\left(\underline{\mu}^{d}\right)^{\mathrm{T}} .
\end{aligned}
$$

Similar to the mean, the covariance does not depend on the velocity. With (13) and (7), the covariance matrix of $\underline{\boldsymbol{d}}$ consists of the single entries $C_{i, j}^{d}$ given by

$$
C_{i, j}^{d}=\mathrm{E}_{\underline{\boldsymbol{x}}_{P}, \underline{\boldsymbol{v}}_{i}, \underline{\boldsymbol{v}}_{j}}\left\{h_{i}\left(\underline{\boldsymbol{x}}_{P}, \underline{\boldsymbol{v}}_{i}\right) \cdot h_{j}\left(\underline{\boldsymbol{x}}_{P}, \underline{\boldsymbol{v}}_{j}\right)\right\}-\mu_{i}^{d} \cdot \mu_{j}^{d} \text {. }
$$

The expected value $\mathrm{E}_{\underline{\boldsymbol{x}}_{P}, \underline{\boldsymbol{v}}_{i}, \underline{\boldsymbol{v}}_{j}}\{\cdot\}$ can be decomposed into nine summands representing the product $h_{i}\left(\underline{\boldsymbol{x}}_{P}, \underline{\boldsymbol{v}}_{i}\right)$. $h_{j}\left(\underline{\boldsymbol{x}}_{P}, \underline{\boldsymbol{v}}_{j}\right)$. In the following, the solution of the expected value $\mathrm{E}_{\underline{\boldsymbol{x}}_{P}, \underline{\boldsymbol{v}}_{i}, \underline{\boldsymbol{v}}_{j}}\{\cdot\}$ for each of the nine summands is derived. The first term is given by

$$
\begin{aligned}
& \mathrm{E}_{\underline{x}_{P}}\left\{\left(\underline{S}_{i}-\underline{\boldsymbol{x}}_{P}\right)^{\mathrm{T}} \cdot\left(\underline{S}_{i}-\underline{\boldsymbol{x}}_{P}\right) \cdot\left(\underline{S}_{j}-\underline{\boldsymbol{x}}_{P}\right)^{\mathrm{T}} \cdot\left(\underline{S}_{j}-\underline{\boldsymbol{x}}_{P}\right)\right\} \\
= & \underline{A}^{\mathrm{T}} \cdot \underline{A} \cdot \underline{B}^{\mathrm{T}} \cdot \underline{B}+\left(\underline{A}^{\mathrm{T}} \cdot \underline{A}+\underline{B}^{\mathrm{T}} \cdot \underline{B}\right) \cdot \operatorname{trace}\left(\mathbf{C}^{x_{P}}\right) \\
& +4 \cdot \underline{A}^{\mathrm{T}} \cdot \mathbf{C}^{x_{P}} \cdot \underline{B}+\left(\operatorname{trace}\left(\mathbf{C}^{x_{P}}\right)\right)^{2}+ \\
& 2 \cdot \underline{1}_{M}^{\mathrm{T}} \cdot\left(\mathbf{C}^{x_{P}} \circ \mathbf{C}^{x_{P}}\right) \cdot \underline{1}_{M},
\end{aligned}
$$


where

$$
\underline{A}=\underline{S}_{i}-\underline{\mu}^{x_{P}}, \underline{B}=\underline{S}_{j}-\underline{\mu}^{x_{P}}
$$

and the operator $\circ$ is the Hadamard (elementwise) product. $\underline{1}_{M}$ is a vector consisting of ones entries, where the variable $M=3$ stands for the three-dimensional space.

In four of the summands, the noise $\underline{v}$ occurs in first or third order and thus the expected value is zero, because the noise process $\underline{\boldsymbol{v}}$ is zero-mean and it is uncorrelated to the target position $\underline{\boldsymbol{x}}_{P}$. The remaining four expected values are given by

$$
\begin{aligned}
& \mathrm{E}_{\underline{\boldsymbol{x}}_{P}, \underline{\boldsymbol{v}}_{j}}\left\{\left(\underline{S}_{i}-\underline{\boldsymbol{x}}_{P}\right)^{\mathrm{T}} \cdot\left(\underline{S}_{i}-\underline{\boldsymbol{x}}_{P}\right) \cdot \underline{\boldsymbol{v}}_{j}^{\mathrm{T}} \cdot \underline{\boldsymbol{v}}_{j}\right\} \\
& =\left(\underline{A}^{\mathrm{T}} \cdot \underline{A}+\operatorname{trace}\left(\mathbf{C}^{x_{P}}\right)\right) \cdot \operatorname{trace}\left(\mathbf{C}_{j}^{v}\right), \\
& \begin{aligned}
\mathrm{E}_{\underline{\boldsymbol{x}}_{P}, \underline{\boldsymbol{v}}_{i}}\left\{\underline{\boldsymbol{v}}_{i}^{\mathrm{T}} \cdot \underline{\boldsymbol{v}}_{i} \cdot\left(\underline{S}_{j}-\underline{\boldsymbol{x}}_{P}\right)^{\mathrm{T}} \cdot\left(\underline{S}_{j}-\underline{\boldsymbol{x}}_{P}\right)\right\} \\
=\left(\underline{B}^{\mathrm{T}} \cdot \underline{B}+\operatorname{trace}\left(\mathbf{C}^{x_{P}}\right)\right) \cdot \operatorname{trace}(\mathbf{C} v), \\
4 \cdot \underline{\mathrm{E}}_{\underline{\boldsymbol{x}}_{P}, \underline{\boldsymbol{v}}_{i}, \underline{\boldsymbol{v}}_{j}}\left\{\left(\underline{S}_{i}-\underline{\boldsymbol{x}}_{P}\right)^{\mathrm{T}} \cdot \underline{\boldsymbol{v}}_{i} \cdot\left(\underline{S}_{j}-\underline{\boldsymbol{x}}_{P}\right)^{\mathrm{T}} \cdot \underline{\boldsymbol{v}}_{j}\right\} \\
=4 \cdot\left(\underline{B}^{\mathrm{T}} \cdot \mathbf{C}_{i, j}^{v} \cdot \underline{A}+\underline{1}_{M}^{\mathrm{T}} \cdot\left(\mathbf{C}^{x_{P}} \circ \mathbf{C}_{i, j}^{v}\right) \cdot \underline{1}_{M}\right),
\end{aligned}
\end{aligned}
$$

and

$$
\begin{aligned}
& \mathrm{E}_{\underline{\boldsymbol{v}}_{i}, \underline{\boldsymbol{v}}_{j}}\left\{\underline{\boldsymbol{v}}_{i}^{\mathrm{T}} \cdot \underline{\boldsymbol{v}}_{i} \cdot \underline{\boldsymbol{v}}_{j}^{\mathrm{T}} \cdot \underline{\boldsymbol{v}}_{j}\right\} \\
& =\operatorname{trace}\left(\mathbf{C}_{i}^{v}\right) \cdot \operatorname{trace}\left(\mathbf{C}_{j}^{v}\right)+2 \cdot \underline{1}_{M}^{\mathrm{T}} \cdot\left(\mathbf{C}_{i, j}^{v} \circ \mathbf{C}_{i, j}^{v}\right) \cdot \underline{1}_{M} .
\end{aligned}
$$

Plugging the results of the five non-zero expected values (15)-(19) into (14), the entry $i, j$ of the covariance matrix $\mathrm{C}^{d}$ is given by

$$
\begin{aligned}
C_{i, j}^{d}= & 4 \cdot \underline{A}^{\mathrm{T}} \cdot \mathbf{C}^{x_{P}} \cdot \underline{B}+2 \cdot \underline{1}_{M}^{\mathrm{T}} \cdot\left(\mathbf{C}^{x_{P}} \circ \mathbf{C}^{x_{P}}\right) \cdot \underline{1}_{M} \\
& +4\left(\underline{B}^{\mathrm{T}} \cdot \mathbf{C}_{i, j}^{v} \cdot \underline{A}+\underline{1}_{M}^{\mathrm{T}} \cdot\left(\mathbf{C}^{x_{P}} \circ \mathbf{C}_{i, j}^{v}\right) \cdot \underline{1}_{M}\right) \\
& +2 \cdot \underline{1}_{M}^{\mathrm{T}} \cdot\left(\mathbf{C}_{i, j}^{v} \circ \mathbf{C}_{i, j}^{v}\right) \cdot \underline{1}_{M},
\end{aligned}
$$

which can be simplified to

$$
C_{i, j}^{d}=4 \cdot \underline{A}^{\mathrm{T}} \cdot \mathbf{C}_{i, j}^{\prime} \cdot \underline{B}+2 \cdot \underline{1}_{M}^{\mathrm{T}} \cdot\left(\mathbf{C}_{i, j}^{\prime} \circ \mathbf{C}_{i, j}^{\prime}\right) \cdot \underline{1}_{M},
$$

if the covariance matrix $\mathbf{C}_{i, j}^{\prime}=\mathbf{C}^{x_{P}}+\mathbf{C}_{i, j}^{v}$ is used.

3) Cross-Covariance: Finally, the cross-covariance

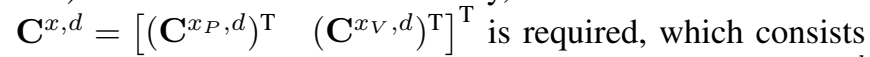
of the cross-covariance depending on the position $\mathbf{C}^{x_{P}, d}$ and the velocity $\mathbf{C}^{x_{V}, d}$. Similar to the covariance $\mathbf{C}^{d}$, the cross-covariance $\mathbf{C}^{x, d}$ is calculated by

$$
\begin{aligned}
\mathbf{C}^{x, d} & =\mathrm{E}_{\underline{\boldsymbol{x}}, \underline{\boldsymbol{d}}}\left\{\left(\underline{\boldsymbol{x}}-\underline{\mu}^{p}\right) \cdot\left(\underline{\boldsymbol{d}}-\underline{\mu}^{d}\right)^{\mathrm{T}}\right\} \\
& =\iiint\left(\underline{x}-\underline{\mu}^{p}\right) \cdot\left(\underline{d}-\underline{\mu}^{d}\right)^{\mathrm{T}} \cdot f(\underline{x}, \underline{d}, \underline{v}) \mathrm{d} \underline{x} \mathrm{~d} \underline{v} \mathrm{~d} \underline{d} \\
& =\iint \underline{x} \cdot \underline{h}(\underline{x}, \underline{v})^{\mathrm{T}} \cdot f(\underline{x}) \cdot f(\underline{v}) \mathrm{d} \underline{x} \mathrm{~d} \underline{v}-\underline{\mu}^{p} \cdot\left(\underline{\mu}^{d}\right)^{\mathrm{T}} \\
& =\mathrm{E}_{\underline{\boldsymbol{x}}, \underline{\boldsymbol{v}}}\left\{\underline{\boldsymbol{x}} \cdot \underline{h}(\underline{\boldsymbol{x}}, \underline{\boldsymbol{v}})^{\mathrm{T}}\right\}-\underline{\mu}^{p} \cdot\left(\underline{\mu}^{d}\right)^{\mathrm{T}} .
\end{aligned}
$$

For calculating the cross-covariance $\mathbf{C}^{x_{P}, d}$ of the position, (20) is used. The cross-covariance for the $i$ th column is calculated by

$$
\begin{aligned}
\underline{C}_{i}^{x_{P}, d} & =\mathrm{E}_{\underline{\boldsymbol{x}}_{P}, \underline{\boldsymbol{v}}_{i}}\left\{\underline{\boldsymbol{x}}_{P} \cdot h_{i}\left(\underline{\boldsymbol{x}}_{P}, \underline{\boldsymbol{v}}_{i}\right)\right\}-\underline{\mu}^{x_{P}} \cdot \mu_{i}^{d} \\
& =-2 \cdot \mathbf{C}^{x_{P}} \cdot \underline{A} .
\end{aligned}
$$

In analogy, the cross-covariance $\mathbf{C}^{x_{V}, d}$ of the velocity is given by

$$
\begin{aligned}
\underline{C}_{i}^{x_{V}, d} & =\mathrm{E}_{\underline{\boldsymbol{x}}_{P}, \underline{\boldsymbol{x}}_{V}, \underline{\boldsymbol{v}}_{i}}\left\{\underline{\boldsymbol{x}}_{V} \cdot h_{i}\left(\underline{\boldsymbol{x}}_{P}, \underline{\boldsymbol{v}}_{i}\right)\right\}-\underline{\mu}^{x_{V}} \cdot \mu_{i}^{d} \\
& =\mathbf{C}^{x_{V}, x_{P}} \cdot\left(\mathbf{C}^{x_{P}}\right)^{-1} \cdot \underline{C}_{i}^{x_{P}, d} \\
& =-2 \cdot \mathbf{C}^{x_{V}, x_{P}} \cdot \underline{A} .
\end{aligned}
$$

The resulting cross-covariance for position and velocity is given for the $i$ th measurement by

$$
\underline{C}_{i}^{x, d}=-2 \cdot \mathbf{C}^{p} \cdot\left[\begin{array}{l}
\mathbf{I}_{M, M} \\
\mathbf{0}_{M, M}
\end{array}\right] \cdot \underline{A},
$$

where $\mathbf{I}_{M, M}$ is the identity matrix and $\mathbf{0}_{M, M}$ is a zero matrix.

\section{Putting It All Together}

The proposed algorithm makes use of a state estimator for recursively calculating the position and the velocity of the object. A state estimator consists of two steps, the prediction and filter step as described above. In the following, a short wrap-up for the filter step is given in vector-matrix notation allowing for a straightforward implementation.

The result of the filter step provides an estimate for the mean $\mu^{e}$ and covariance matrix $\mathbf{C}^{e}$ according to (9). In doing so, the unknown moments $\underline{\mu}^{d}, \mathbf{C}^{d}$, and $\mathbf{C}^{x, d}$ are given by

$$
\begin{aligned}
\underline{\mu}^{d}= & (\mathbf{H} \circ \mathbf{H})^{\mathrm{T}} \cdot \underline{1}_{M}+\underline{1}_{N} \cdot \operatorname{trace}\left(\mathbf{P} \cdot \mathbf{C}^{p} \cdot \mathbf{P}^{\mathrm{T}}\right) \\
& +\mathbf{K}^{\mathrm{T}} \cdot \operatorname{diag}\left(\mathbf{C}^{v}\right), \\
\mathbf{C}^{x, d}= & -2 \cdot \mathbf{C}^{p} \cdot \mathbf{P}^{\mathrm{T}} \cdot \mathbf{H}, \\
\mathbf{C}^{d}= & \mathbf{K}^{\mathrm{T}} \cdot\left(4 \cdot\left(\operatorname{vec}(\mathbf{H}) \cdot \operatorname{vec}(\mathbf{H})^{\mathrm{T}}\right) \circ \mathbf{T}+2 \cdot \mathbf{T} \circ \mathbf{T}\right) \cdot \mathbf{K},
\end{aligned}
$$

with

$$
\begin{aligned}
\mathbf{S} & =\left[\begin{array}{lll}
\underline{S}_{1} & \ldots & \underline{S}_{N}
\end{array}\right], \mathbf{P}=\left[\begin{array}{ll}
\mathbf{I}_{M, M} & \mathbf{0}_{M, M}
\end{array}\right], \\
\mathbf{H} & =\mathbf{S}-\left(\underline{1}_{N}\right)^{\mathrm{T}} \otimes\left(\mathbf{P} \cdot \underline{\mu}^{p}\right), \mathbf{K}=\mathbf{I}_{N, N} \otimes \underline{1}_{M}, \\
\mathbf{T} & =\mathbf{C}^{v}+\mathbf{1}_{N, N} \otimes\left(\mathbf{P} \cdot \mathbf{C}^{p} \cdot \mathbf{P}^{\mathrm{T}}\right),
\end{aligned}
$$

where $\otimes$ is the Kronecker product, $\operatorname{vec}(\mathbf{H})$ is the vector-version of the matrix $\mathbf{H}$, and $\mathbf{1}_{N, N}$ is a one matrix. The vector-version is defined as $\operatorname{vec}(\mathbf{H})=$ $\left[\begin{array}{lll}\left(\underline{H}_{1}\right)^{\mathrm{T}} & \ldots & \left(\underline{H}_{N}\right)^{\mathrm{T}}\end{array}\right]^{\mathrm{T}}$. The variable $M=3$ stands for the three-dimensional space and $N$ for the number of landmarks. Furthermore, the measured ranges $\underline{\hat{r}}$ have to be squared according to $\underline{\hat{d}}=\underline{\hat{r}} \circ \underline{\hat{r}}$.

\section{Computational Complexity}

In order to calculate the required moments $\mu^{d}, \mathbf{C}^{d}$, and $\mathbf{C}^{x, d}$ for the filter step, the computational complexity for summation and multiplication for the proposed approach is in $\mathrm{O}\left(N^{2} \cdot M^{2}\right)$. Compared to the proposed approach the computational complexity for calculating the matrix root in the Unscented Kalman Filter is in $\mathrm{O}\left(N^{3} \cdot M^{3}\right)$. 


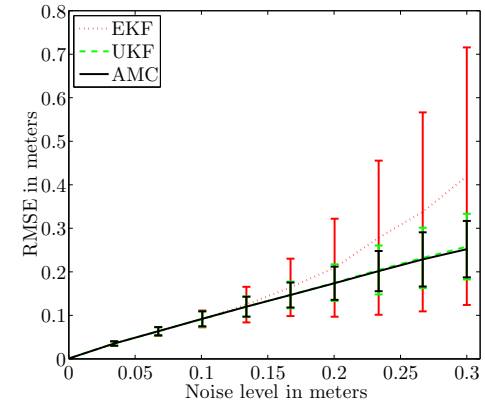

(a) Simulation results for the three estimators AMC, UKF, and EKF.

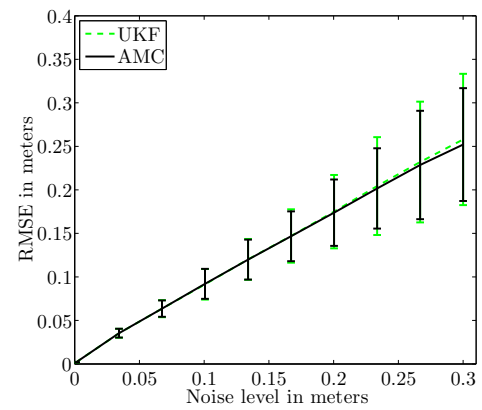

(b) Only the results of AMC and UKF.

Fig. 1. Simulation results for the three estimators AMC, UKF, and EKF. The average RMSE and its standard deviation for different noise levels.

\section{EXPERIMENTS}

In this section, simulations and real-world experiments are used for comparing the proposed approach (AMC) with standard state estimators like the Extended Kalman Filter (EKF) and the Unscented Kalman Filter (UKF). Furthermore, a closed-form solution (CFS) [3] is considered for the real world experiment. All three estimators (AMC, EKF, and $\mathrm{UKF}$ ) make use of the measurement equation (4) and the system model (1).

\section{A. Simulation}

To compare the performance of the three algorithms (AMC, EKF, UKF), range measurements to four landmarks with positions

$$
\mathbf{S}=\left[\begin{array}{lll}
\underline{S}_{1} & \ldots & \underline{S}_{4}
\end{array}\right]=\left[\begin{array}{cccc}
-2 & -2 & 2 & 2 \\
-2 & 2 & -2 & 2 \\
0 & 0 & 0 & 2
\end{array}\right] \mathrm{m}
$$

are performed. For generating the noisy range measurements, ten different noise levels $\mathbf{C}_{i}^{v}=\mathbf{I}_{3,3} \cdot \sigma_{n}^{2}$ for each landmark $i=1, \ldots, 4$ are considered, where $\sigma_{n}=\frac{n-1}{30} \mathrm{~m}$ and $n=1, \ldots, 10$. For each noise level, 1000 random object trajectories are generated. Each trajectory consists of 100 measurements to all landmarks.

The Gaussian density representing the initial state at time step $k=0$ has zero mean and an initial covariance, which is $\mathbf{C}_{0}=\operatorname{diag}\left(\left[\begin{array}{ll}10 \cdot \underline{1}_{3}^{\mathrm{T}} & 10 \cdot \underline{1}_{3}^{\mathrm{T}}\end{array}\right]\right)$. The sampling time is $T=$ 0.1 seconds. The process noise is $C_{c, x}^{w}=0.01, C_{c, y}^{w}=$ 0.01 , and $C_{c, z}^{w}=0.0001$.

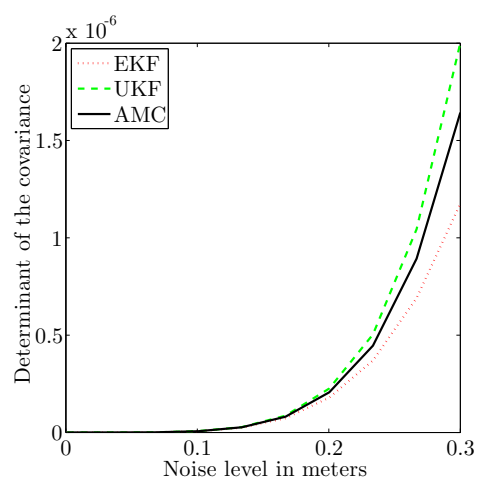

(a) Mean of the determinant from the covariance matrix for all test runs at each noise level for AMC, UKF, and EKF.

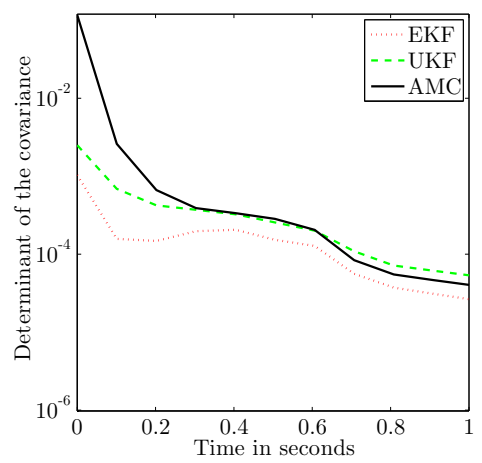

(b) Determinant for one single run at the noise level $0.3 \mathrm{~m}$.

Fig. 2. Determinant of the covariance matrix from AMC, UKF, and EKF at different noise levels.

For each random trajectory, the Root Mean Square Error (RMSE) between the estimate and the ground truth is calculated. In Fig. 1, the average RMSE and its standard deviation over the 1000 test runs for all the different noise levels is shown.

For small noise, all three estimators perform similar. If the noise increases, the RMSE of the EKF increases much stronger compared to the other two estimators. For a high noise level, the UKF and the proposed approach present comparable results, where the average RMSEs and the standard deviations of the AMC are slightly smaller.

All three estimators have to evaluate (5), but compared to $\mathrm{AMC}$ and $\mathrm{EKF}$, the UKF additionally requires matrix roots for determining the sigma points. Consequently, the computational demand of the UKF is much higher. For calculating the required moments in (21), only vector-matrix products and no additional matrix inversions or roots are required. Of course, the complexity of the EKF is lower compared to the AMC, but for a high noise level, the AMC performs significantly better.

The average determinant of the covariance matrix of the position estimate $\underline{\boldsymbol{x}}_{k, P}$ of all test runs is shown in Fig. 2 (a). In Fig. 2 (b), one single test run at noise level $0.3 \mathrm{~m}$ exemplarily demonstrates the evolution of the determinant of the covariance over the time. It can be seen that the covariance of the EKF decreases too quickly. Due to the 


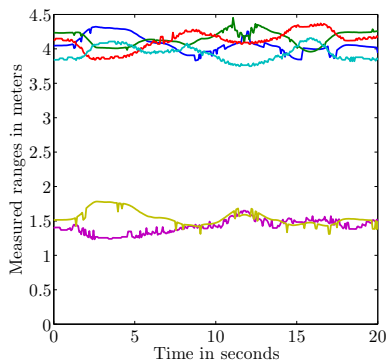

(a) Measured ranges between one (b) Estimated trajectories of the microphone and six landmarks.

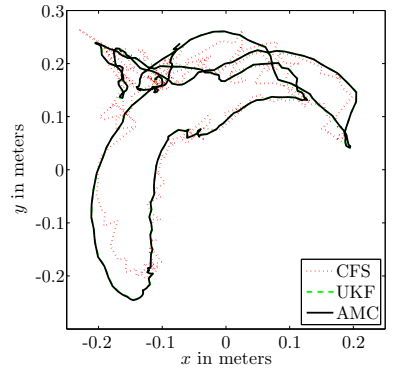

$\mathrm{AMC}, \mathrm{UKF}$, and CFS.
Fig. 3. The measured ranges and estimated trajectories.

linearization based on first-order Taylor-series expansions, the determinant of the EKF is too small and thus the EKF is too certain about its estimate. Hence, the estimation results are inconsistent, which is often a problem when using an EKF. On the other hand, sample-based approaches as the UKF or analytic approaches as the AMC overcome this problem. The determinant of the AMC is smaller compared to the determinant of the UKF. Furthermore, as described before, the RMSE of the AMC is smaller as well. All together, the AMC is more informative compared to the UKF.

\section{B. Experiment}

The experiment considers a tracking system for extended range telepresence [12] for generating the range data. Six loudspeakers emit signals that are received by four microphones attached to the object. Based on the emitted and received signals, ranges between each microphone and each loudspeaker are calculated. The measured ranges for one microphone are shown in Fig. 3(a).

The initial mean is zero and the initial covariance is $\mathbf{C}_{0}=\operatorname{diag}\left(\left[\begin{array}{cc}10 \cdot \underline{1}_{3}^{\mathrm{T}} & 10 \cdot \underline{1}_{3}^{\mathrm{T}}\end{array}\right]\right)$. The process noise was set to $C_{c, x}^{w}=0.04, C_{c, y}^{w}=0.04$, and $C_{c, z}^{w}=0.01$. The sampling time is $T=0.0625$ seconds. The standard deviation of the measurement noise is assumed to be $0.1 \mathrm{~m}$.

It can be seen in Fig. 3(b) that a closed-form solution as in [3] provides poor results, if the range measurements are noisy. On the other hand, state estimators like the AMC or UKF can deal with noisy range measurements. The results of the state estimators (AMC, UKF) are similar, since the noise level is small (please recall Sec. V-A). The average of the absolute position error of all microphone pairs is $0.0336 \mathrm{~m}$ for the AMC, $0.0337 \mathrm{~m}$ for the UKF, $0.0342 \mathrm{~m}$ for the EKF, and $0.0423 \mathrm{~m}$ for the closed-form solution (CFS). Even if the AMC merely provides a slightly better result than the UKF and the EKF, this experiment demonstrates that the AMC also works in a real-world scenario.

\section{CONCLUSIONS}

This paper presents a Bayesian state estimator for rangebased localization. Assuming that the object's state estimate is Gaussian, the required moments, i.e., mean and covariance are calculated analytically. Compared to well-known closedform solutions assuming exact (or almost exact) range measurements, the proposed approach allows taking subspace measurements into account. Furthermore, AMC facilitates dynamic localization and provides information about the object's position and velocity uncertainty.

Due to the assumption that state and measurement are jointly Gaussian, AMC provides an optimal stochastic linearization, which takes the entire Gaussian density into account. Other Gaussian estimators based on linearization merely consider points, i.e., EKF uses first-order Taylorseries expansion around the mean and the UKF calculates sigma-points for stochastic linearization. As demonstrated in the simulations and experiments, AMC leads to more accurate and more consistent localization results compared to the EKF or UKF. The computational demand of AMC is lower than that of the UKF, because no matrix roots have to be calculated. Furthermore, no parameter adaption for sigmapoint calculation is necessary.

It is intended to utilize the analytic expressions for the mean and covariance for improving existing results in research fields such as sensor placement and scheduling or simultaneous localization and mapping (SLAM).

\section{REFERENCES}

[1] F. Seco, A. Jimenez, C. Prieto, J. Roa, and K. Koutsou, "A Survey of Mathematical Methods for Indoor Localization," in IEEE International Symposium on Intelligent Signal Processing (WISP 2009), Aug. 2009, pp. 9-14.

[2] J. J. Caffery, "A New Approach to the Geometry of TOA Location," in Proceedings of 55th IEEE Vehicular Technology Conference, 2000, pp. 1942-1949.

[3] U. D. Hanebeck and G. Schmidt, "Closed-Form Elliptic Location with an Arbitrary Array Topology," in Proceedings of IEEE Intl. Conference on Acoustics, Speech, and Signal Processing, 1996, pp. 3070-3073.

[4] F. Thomas and L. Ros, "Revisiting Trilateration for Robot Localization," IEEE Transactions on Robotics, vol. 21, no. 1, pp. 93-101, Feb. 2005.

[5] D. E. Manolakis, "Efficient Solution and Performance Analysis of 3-D Position Estimation by Trilateration," IEEE Transactions on Aerospace and Electronic Systems, vol. 32, no. 4, pp. 1239-1248, Oct. 1996.

[6] K. Cheung and H. C. So, "A Multidimensional Scaling Framework for Mobile Location Using Time-of-Arrival Measurements," IEEE Transactions on Signal Processing, vol. 53, no. 2, pp. 460-470, Feb. 2005.

[7] S. F. Schmidt, Advances in Control Systems, Volume 3. Academic Press, New York, 1966, ch. Application of State-Space Methods to Navigation Problems, pp. 293-340.

[8] S. J. Julier and J. K. Uhlmann, "Unscented Filtering and Nonlinear Estimation," Proceedings of the IEEE, vol. 92, no. 3, pp. 401-422, 2004.

[9] G. Welch, B. D. Allen, A. Ilie, and G. Bishop, "Measurement Sample Time Optimization for Human Motion Tracking/Capture Systems," in Proceedings of Trends and Issues in Tracking for Virtual Environments, Workshop at the IEEE Virtual Reality 2007 Conference, 2007.

[10] M. Clark and R. Vinter, "A New Class of Moment Matching Filters for Nonlinear Tracking and Estimation Problems," in Nonlinear Statistical Signal Processing Workshop, 2006 IEEE, Sept. 2006, pp. 108-112.

[11] A. Papoulis and S. U. Pillai, Probability, Random Variables and Stochastic Processes. McGraw-Hill Science/Engineering/Math; 4. Edition, 2002.

[12] P. Rößler, F. Beutler, U. D. Hanebeck, and N. Nitzsche, "Motion Compression Applied to Guidance of a Mobile Teleoperator," in Proceedings of the 2005 IEEE International Conference on Intelligent Robots and Systems (IROS 2005), 2005, pp. 2495-2500. 